\title{
L. A study of an apparatus for the determination of the rate of diffusion of solids dissolved in liquids
}

\author{
Albert Griffiths M.Sc.(Vic.) A.R.C.S. (Lond.)
}

To cite this article: Albert Griffiths M.Sc.(Vic.) A.R.C.S. (Lond.) (1899) L. A study of an apparatus for the determination of the rate of diffusion of solids dissolved in liquids, Philosophical Magazine Series 5, 47:289, 530-539, DOI: 10.1080/14786449908621294

To link to this article: http://dx.doi.org/10.1080/14786449908621294

曲 Published online: 08 May 2009.

Submit your article to this journal $₫$

ЏII Article views: 2

Q View related articles $匚$ 


\section{[ 530 ]}

L. A Study of an Apparatus for the Determination of the Rate of Thiffusion of Solids dissolved in Liquids. By Alber'T Grifeiths, M.Sc.(Vic.), A.R.C.S.(Lond.)**.

\section{Section I.}

$\mathrm{F}^{\mathrm{OR}}$ some time the author has been engaged in some experiments with the object of determining the coefficient of diffusion of bodies dissolved in water. It may be some years before experimental results of any decided value are obtained; and perhaps he will be allowed to give an account of the calculations involved, and of the methods he has adopted in obtaining an idea of the probable order of magnitude of the errors to which the apparatus is liable.

Fig. 1.

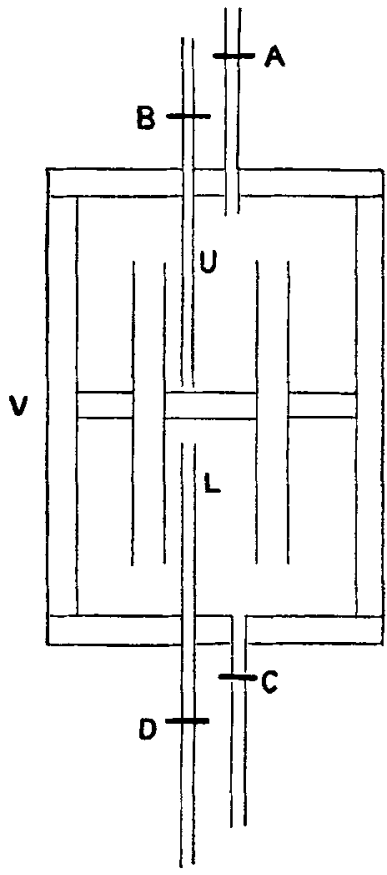

The apparatus consists of a vessel $\mathrm{V}$, divided into two compartments, $U$ and $L$, by a diaphragm through which pass a number of equal vertical tubes of which two only are shown in the figure. Two tubes, A, B, provided with stop-cocks, pass into U. One, A, just enters U ; the other passes down to the? bottom. Similarly two tubes (? and $\mathrm{D}$ pass into $\mathrm{L}$; $\mathrm{C}$ just enters, and $\mathrm{D}$ reaches to the top. The vessel is first completely filled with water, and in the case of a substance which produces an aqueous solution with a specific gravity greater than unity, the tubes $A$ and $B$ are closed, and the solution is passed in through $\mathrm{C}$, the water of $\mathrm{L}$ being allowed to escape through D. Diffusion along the vertical tubes now commences, and the compartments $U$ and $L$ are alternately and periodically refilled with pure water and solution respectively. A quantitative analysis is made of the liquid taken from the upper compartment.

Snction II.-Calculation of the Coefficient of Diffusion.

Lord Kelvin has solved the problem of the flow of electricity along a cable possessing appreciable capacity. This

* Communicated by the Physical Society : read Feb. 24, 1899. 
Determination of Diffusion of Solids dissolved in Liquids. $\quad 531$ solution can readily be transferred to the problem under consideration.

It will be assumed that the upper extremities of the tubes are kept in contact with pure water, and the lower extremities in contact with a solution of constant strength.

Let $\gamma=$ quantity of dissolved substance which enters the upper compartment in unit time, when the combined sectional area of the tubes equals unity.

$k=$ coefficient of diffusion (C.G.S. system), assumed to be a constant.

$\mathrm{L}=$ length of tubes in centims.

$\mathbf{T}=$ quantity of substance per c. c. in the lower compartment.

$t=$ time in seconds.

Then

$$
\gamma=\frac{k \mathrm{~T}}{\mathrm{~L}}\left\{1+2 \sum_{i=1}^{i=\infty}(-1)^{i} e^{\frac{-i^{i} \pi^{2} k t}{\mathrm{~L}^{2}}}\right\},
$$

where $i$ is any integer.

[If $\frac{\mathrm{L}^{2}}{\pi^{2} k} \log \frac{4}{3}$ be taken as the unit of time, the topmost of the series of curves given on page 72, vol, ii., of Kelvin's 'Mathematical and Physical Papers,' shows graphically how $\gamma$ rises to a maximum.]

Let $q=$ total quantity of substance transmitted in $t$ seconds. Integrating the preceding expression with respect to $t$, we obtain

$$
q={ }_{\mathrm{L}}^{k \mathrm{~T}}\left\{t+2 \sum_{i=1}^{i=\infty}(-1)^{i} \frac{\mathrm{L}^{2}}{i^{2} \pi^{2} k}-2 \sum_{i=1}^{i=\infty}(-1)^{i} \frac{\mathrm{L}^{2}}{i^{2} \pi^{2} k} e^{\frac{-i^{2} \pi^{2} k t}{\mathrm{~L}^{2}}}\right\}
$$

When $\mathrm{t}$ is very large the third term within the brackets may be neglected, and

$$
q=\frac{k \mathrm{~T} t}{\mathrm{~L}}+\frac{2 \mathrm{LT} T}{\pi^{2}} \sum_{i=1}^{i=\infty} \frac{(-1)^{i}}{i^{2}} \quad . \quad . \quad .
$$

or

$$
q=\frac{k \mathrm{~T} t}{\mathrm{~L}}-\frac{2 \mathrm{LT}}{\pi^{2}} \frac{\pi^{2}}{12}=\frac{k \mathrm{~T} t}{\mathrm{~L}}-\frac{\mathrm{LT}}{6} . . .
$$

The last equation shows that if $t$ is large the quantity transmitted equals $k \mathrm{~T} t / \mathrm{L}$ minus a quantity which is independent of the coefficient of diffusion. Thus if in the adjoining tigure abscissæe represent times, and ordinates the quantities 
532 Mr. A. Griffiths on an Apparatus for the Determination diffused per second, the shaded area between the asymptote and the curve is independent of the coefficient of diffusion.

Fig. 2.

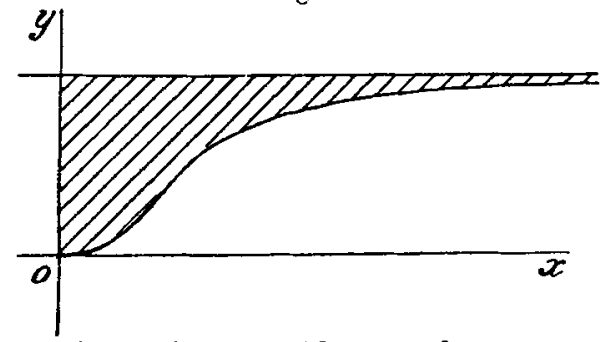

The table below gives an idea of the rate at which the steady state is attained when $\mathrm{L}=4.043$, and $k=3 \times 10^{-6}$. The unit of quantity is the amount transmitted per week on the attainment of the steady state.

\begin{tabular}{|c|c|c|}
\hline No. of Weeks. & Total Quantity. & Quantity per Week. \\
\hline $1 \ldots \ldots$ & $0 \cdot 104$ & $0 \cdot 104$ \\
\hline $2 \ldots$ & 0.703 & 0.599 \\
\hline $3 \ldots \ldots$ & $1 \cdot 567$ & 0.864 \\
\hline $4 \ldots$ & $2 \cdot 519$ & 0.952 \\
\hline $5 \ldots \ldots$ & $3 \cdot 505$ & 0.986 \\
\hline $6 \ldots \ldots$ & $4 \cdot 500$ & 0.995 \\
\hline $7 \ldots$ & $5 \cdot 498$ & \\
\hline $8 \ldots \ldots$. & $6 \cdot 498$ & \\
\hline $9 \ldots .$. & $7 \cdot 498$ & \\
\hline $10 \ldots \ldots$ & $8 \cdot 498$ & \\
\hline
\end{tabular}

[The above table gives the integral quantities transmitted. The curve already mentioned shows the way in which the rate of transmission varies. The unit abscissa $\frac{\mathrm{L}^{2}}{\pi^{i} k} \log _{\odot} \frac{4}{3}=$
2626 of a week.]

\section{Section III.-Error due to Differences of Temperature.}

The object of this section is to obtain the order of magnitude of the errors produced by differences in temperature between the various tubes. 
of the Rate of Diffusion of Solids dissolved in Liquids. $\quad 533$

For simplicity, two tubes only will be considered whose lengths and sectional areas are the same. The expansion cf the material of the tubes will be neglected. To make the problem amenable to mathematical treatment, it will be assumed that the density of water equals $(1-\alpha \theta)$, where $\theta$ is the temperature, and $\alpha$ is approximately equal to an ideal coefficient of expansion. It will also be assumed that

$$
d=(1-\alpha \theta+t),
$$

where

$$
d=\text { the density of the solution, }
$$

$$
t=\text { the concentration. }
$$

Let $\theta_{1}=$ the temperature of one tube.

$v_{1}=$ the velocity of the liquid up the tube.

$\theta_{2}=$ the temperature of the second tube.

$v_{2}=$ the velocity of the liquid up the tube.

$\mathrm{L}=$ the length of each tube, assumed constant.

$k=$ the coefficent of diffusion, assumed constant.

$\mathrm{T}=$ the concentration of the solution at the bottom of each tube.

From considerations similar to those adopted in "Diffusive Convection "*, Sect. II. and III., it can be shown that, neglecting viscosity,

$$
\begin{aligned}
& g\left(1-\alpha \theta_{1}\right) \mathrm{L}+\frac{g \mathrm{TL}}{1-e^{-\frac{v_{1} \mathrm{~L}}{k}}}-\frac{k g \mathrm{~T}}{v_{1}} \\
& \quad=g\left(1-\alpha \theta_{2}\right) \mathrm{L}+\frac{g \mathrm{TL}}{1-e^{-\frac{v_{2} \mathrm{~L}}{k}}}-\frac{k g^{\prime} \mathrm{T}}{v_{2}} .
\end{aligned}
$$

Di viding throughout by $g$, and expanding,

$$
\begin{aligned}
& \left(1-\alpha \theta_{1}\right) \mathrm{L}+\mathrm{TL}\left(\frac{k}{v_{1} \mathrm{~L}}+\frac{1}{2}+\frac{v_{1} \mathrm{~L}}{12 k}+\& \mathrm{c} .\right)-\frac{k \mathrm{~T}}{v_{1}} \\
& =\left(1-\alpha \theta_{2}\right) \mathrm{L}+\mathrm{TL}\left(\frac{k}{v_{2} \mathrm{~L}}+\frac{1}{2}+\frac{1}{12} \frac{v_{2} \mathrm{~T}}{12 k}+\& \mathrm{c} .\right)-\frac{k \mathrm{~T}}{v_{2}} ;
\end{aligned}
$$

and

$$
\left(v_{2}-v_{1}\right)=\frac{12 k \alpha\left(\theta_{2}-\theta_{1}\right)}{\mathrm{TL}}, \text { approx. }
$$

* Phil. Mag. Nov. 1898, pp. 453-465.

Phil. Mag. S. 5. Vol. 47. No. 289. June 1899. 
534 Mr. A. Griffiths on an Apparatus for the Determination

Now

hence

$$
\frac{v_{1}}{v_{2}}=-\left(\frac{1-\alpha \theta_{2}}{1-\alpha \theta_{1}}\right)
$$

and

$$
v_{2}\left\{1+\frac{1-\alpha \theta_{2}}{1-\alpha \theta_{1}}\right\}=\frac{12 k \alpha\left(\theta_{2}-\theta_{1}\right)}{\mathrm{TL}},
$$

$$
\frac{v_{2} \mathrm{~L}}{k}=\frac{12 \alpha\left(1-\alpha \theta_{1}\right)\left(\theta_{2}-\theta_{1}\right)}{\mathrm{T}\left(2-\alpha \bar{\theta}_{1}+\theta_{2}\right)}
$$

similarly

$$
\frac{v_{1} \mathrm{~L}}{k}=\frac{12 \alpha\left(1-\alpha \theta_{2}\right)\left(\theta_{1}-\theta_{2}\right)}{\mathrm{T}\left(2-\alpha \bar{\theta}_{1}+\theta_{2}\right)}
$$

The correcting factor equals

$$
\begin{gathered}
\frac{\left\{1+\frac{1}{2}-\frac{v_{1} \mathrm{~L}}{k}+\frac{1}{12} \frac{v_{1} \mathrm{~L}^{2}}{k^{2}}\right\}+\left\{1+\frac{1}{2} \frac{v_{2} \mathrm{~L}}{k}+\frac{1}{12} \frac{v_{2}{ }^{2} \mathrm{~L}^{2}}{k^{2}}\right\}}{2} \\
=1+\frac{1}{4}\left(\frac{v_{1} \mathrm{~L}}{k}+\frac{v_{2} \mathrm{~L}}{k}\right)+\frac{1}{24}\left(\frac{v_{1}{ }^{2} \mathrm{~L}^{2}}{k^{2}}+\frac{v_{2}{ }^{2} \mathrm{~L}^{2}}{k^{2}}\right) \\
=1+\frac{3 \alpha^{2}\left(\theta_{2}-\theta_{1}\right)^{2}}{\mathrm{~T}}+\frac{3 \alpha^{2}\left(\theta_{2}-\theta_{1}\right)^{2}}{\mathrm{~T}^{2}}, \text { approx. }
\end{gathered}
$$

As an example, if $\left(\theta_{2}-\theta_{1}\right)=0^{\sigma_{1}} 1\left(\mathrm{C}, \mathrm{T}=0^{-1}\right.$, and $\alpha=0.00015$, the correcting factor equals $1 \cdot 00000007$.

When $T$ is a small fraction of nnity, as in general will be the case, the correcting factor equals

$$
1+\frac{3 \alpha^{2}\left(\theta_{2}-\theta_{1}\right)^{2}}{\mathrm{I}^{2}}, \text { approx. }
$$

It may be noted that, approximately,

$$
v_{2}=-v_{1}=\frac{12 k \alpha\left(\theta_{2}-\theta_{2}\right)}{\mathrm{TL}} \text {. }
$$

As an example, let $k=3 \times 10^{-6}, \alpha=0.00015,\left(\theta_{2}-\theta_{k}\right)=0.1$, $\mathrm{T}=0 \cdot 1, \mathrm{~L}=4 \mathrm{~cm}$.

Then

$$
\begin{aligned}
v_{2} & =1.35 \times 10^{-9} \mathrm{~cm} . \text { per second, } \\
& =0.04 \mathrm{~cm} . \text { per year. }
\end{aligned}
$$


of the Rate of Diffusion of Solids dissolced in Liquidl. 50.5

Secrion IV.-Errors due to Changes of Temperature of Apparatus as a whole.

Apart from the effects of any differences of temperature which may exist between the tubes themselves, there will in general be a flow set up along them one way or the other when the apparatus as a whole is heated.

If the upper and lower compartments were of equal volume and the coefficients of expansion and the compressibilities of water and solution were the same, there wonld be no movement along the tubes; the liquid would simply be compressed slightly. The actual motion conid only be calculated from a knowledge of the various coefficients of expansion and the compressibilities. The author has not the dati for an aceurate calculation, but to give an idea of the order of magnitude of the error, the case will be considered in which the taps of the upper compartment are open.

Let $\quad T=$ volume of lower compartment.

$\alpha=$ coefficient of cubical expansion of liquid.

$\theta=$ rise in temperature per second.

It will be assumed that the temperature increases at a constant rate, and that the steady state has been attained.

Let $\quad v=$ velocity $u p$ the tubes per second.

$\mathrm{L}=$ length of each tube.

Then $\quad v=\frac{\mathrm{V} \alpha \theta}{\mathrm{A}}$.

The quantity of dissolved substance transmitted per second (see Diffusive Convection, Sec. IV.) equals, when VL/k is small,

$$
\begin{array}{r}
\frac{k \mathrm{AT}}{\mathrm{L}}\left(1+\frac{1}{2} \frac{v \mathrm{~L}}{k}\right) \text { approx., } \\
\text { i. e. } \frac{k \mathrm{AT}}{\mathrm{L}}\left(1+\frac{1}{2} \frac{\mathrm{VLa} \theta}{\mathrm{A} k}\right) .
\end{array}
$$

As an example, let the rise of temperature be $2^{\circ} \mathrm{C}$. per week ; let $a=0.00015, \mathrm{~L}=4 \mathrm{~cm}$., $V=40$ c.c., $A=0 \cdot 4$, and $k=3 \times 10^{-6}$. Then VLa $\mathrm{V} / 2 \mathrm{~A} k$ equals 0.033 ; and the error due to the flow produced by the expansion is more than 3 per cent.

It may be mentioned that if a bubble of air is present in the lower compartment, it increases the flow, and the corresponding error, considerably.

Even if the upper taps are not left continuously open, there is an error corresponding to the above; for opening 
$536 \mathrm{Mr}$. A. Griffiths on an Apparatus for the Determination the top taps will allow a sudden rush to take place up the tubes.

It is obvious that a diminution of temperature produces an error in the opposite direction; and the error produced by a fluctuating temperature will probably not be considerable in the long run.

Section V.-Errors due to Changes in Volume produced by the Weakening of the Solution through Diffusion.

In general, when a solution loses some of the dissolved substance by diffusion it diminishes in volume. The diminution depends on the nature of the dissolved substance and varies with the concentration. The diminution for any given loss can be calculated from a table of densities. For example, an aqueous solution of copper sulphate at a temperature of $23^{\circ} \cdot 3$ containing 80 grams to the litre has a density of $1 \cdot 080$, whereas a solution containing $160 \mathrm{grams}$ to the litre has a density of $1 \cdot 154$. It can be readily shown that the addition of 1 gram of anhydrous copper sulphate, between the given limits, on the average increases the volume by 0.075 c.c. approx.

As in Section IV. it will be assumed that the upper compartment is open to the atmosphere.

Since the diminution of volume of the lower compartment equals the quantity of copper sulphate transmitted multiplied by 0.075 , with the aid of Sec. I. "Diffusive Convection," it can readily be seen that

$$
v=\frac{-1 \cdot 075 \times \mathrm{T} \times v}{1-e^{-\frac{v \mathrm{~L}}{k}}}
$$

Hence, neglecting the second and higher powers of $\frac{v \mathrm{~L}}{k}$,

$$
\frac{v \mathrm{~L}}{k}=0.075 \times \mathrm{T} \text {. }
$$

Let

$$
\mathrm{T}=0 \cdot 12 \text {, then } \frac{v \mathrm{~L}}{k}=0 \cdot 009 .
$$

The fractional diminution in the quantity transmitted equals $v \mathrm{~L} / 2 k$, i.e. $0 \cdot 0045$. Thus the motion along the tubes causes a diminution in the quantity transmitted by about one half per cent.

With salts whose solutions experience greater changes in volume, the error may be very appreciable.

'Thus, between the limits of density 1.081 and $1 \cdot 159$, the addition of 1 gram of sodium chloride causes an average 
of the Rate of Diffusion of Solids dissolved in Liguids. 537 increase in volume of 0.35 c.c.; and with $\mathrm{T}=0.12$, the error would be $2 \cdot 1$ per cent.

It may be noted that when a small quantity of copper sulphate is added to water, the resultant volume is about the same as the original volume of the water. Hence if the lower compartment were left open to the atmosphere instead of the upper one, there would be no error from the cause under consideration.

As before errors are produced when the taps are opened intermittently.

SECTION VI.-Errors due to Circulations produced when the Liquids of the Upper and Lower Compartments are renewed.

When the water in the upper compartment is renewed the pressure at the inlet is greater than the pressures at the outlet, and in consequence the pressures at the upper extremities of the tubes are not constant. Hence flows take place up certain tubes and down others.

Let there be $n$ tubes; and let $p_{1}, p_{2}, \& c$. be the respective excesses of the pressures at the tops of the tubes above the average pressure. Assuming the attainment of the steady state, and making the usual approximations, it can readily be shown that the correcting factor equals

$$
1+\frac{12}{g^{2} T^{2} L^{2}}\left(\frac{p_{1}{ }^{2}+\ldots p_{n}{ }^{2}}{n}\right) \text {. }
$$

Let $p_{r}$ be the greatest of the $p$ 's ; then the fractional error is loss than $12 p_{r}^{2} / g^{2} T^{2} L^{2}$. Neglecting inertia effects, the value of $p_{r}$ must be less than the difference of pressure between the inlet and outlet, an estimate of which could be made practically. The author has not made any practical measurements, but has made a guess as to the order of magnitude of the difference of pressure by making a crude analogy between the upper compartment and a cylindrical tube. Thus, if 100 c.c. of water pass along a tube $3 \mathrm{~cm}$. in diameter in an hour, the difference of pressure (assuming the formula for a capillary to hold) between two points on the axis 3 centim. apart, if we take the coefficient of viscosity equal to 0.01 , is 0.004 dyne. Let $p_{r}=0.01, \mathrm{~T}=0.1, \mathrm{~L}=4$, then the fructional error equals

$$
12 \times(\cdot 01)^{2} /(981)^{2}(0 \cdot 1)^{2} \times 4^{2} \text {, or } 7 \cdot 8 \times 10^{-8} \text {. }
$$

It is clear that the error from this cause is appreciable. 
538 Determination of Diffusion of Solids dissolved in Liquids.

Sectron VII.-Error due to Diffusive Convection.

Inequalities in the lengths of the tubes and inaccuracies in placing them in the diaphragm will, in general, produce convection-currents. There is no intrinsic difficulty in calculating flows of small magnitude, if the tubes are of unequal length; but, to simplify the algebra, in the following the tubes will be taken of equal length and of equal sectional area.

Let $n=$ number of tubes.

$\delta_{1}=$ distance between the top of one tube and the average of the tops of all the tubes.

$\delta_{r}=$ corresponding distance for the $r$ th tube.

$v_{1}=$ velocity up the first tubo.

$v_{r}=", \quad r$ th tube.

Making the usual assumptions, it can be shown that

$$
\frac{\left(v_{1}-v_{r}\right) \mathrm{L}^{2}}{12 k}=\delta_{r}-\delta_{1} \text {. }
$$

Noting that $\Sigma v_{r}=0, \Sigma \delta_{r}=0$, it can readily be shown that

$$
\frac{v_{r} \mathrm{~L}}{k}=\frac{-12 \delta_{r}}{\mathrm{~L}}
$$

and that the correcting factor equals

$$
1+\frac{12}{L^{2}} \frac{\delta_{1}{ }^{2}+\ldots \delta_{n}^{2}}{n} \text {. }
$$

With ordinary care in the construction of the apparatus, the correcting factor will deviate very little from unity.

Section VIII.-Sume Experimental Results.

One apparatus has 15 tubes, of average length 4.043 centim., and a total sectional area 0.4102 square centim. It was placed in a cellar the temperature of which fluctuated through a range of about $3^{\circ} \mathrm{C}$. per week. The lower compartment contained a solution of copper sulphate holding 0.0506 of copper to the c.c. At intervals of a week the upper compartment was renewed with pure water ; similarly the lower compartment was renewed with fresh solution. 'The tubes were originally full of pure water. The averıge temperature was about $8^{\circ} \mathrm{C}$. By the end of the first week $0.0026 \mathrm{gram}$ of copper had been transmitted; by the end of the second a total of 0.0077 ; end of third, 0.0144 ; end of fourth, 0.0226 ; end of tifth, 0.0311 ; end of sixth, 0.0403 ; end of eighth, 0.0559 ; end of ninth, 0.0662 . 
Taking as a basis the quantity transmitted in nine weeks, the coefficient of diffusion equals $28 \cdot 7 \times 10^{-7}$. This result, and others not recorded, indicate that the errors due to the various causes studied are, at any rate, not enormous.

To diminish the variations of temperature, in one experiment the whole apparatns was put inside a copper cylinder; but, unfortunately, some air-bubbles were accidentally allowed to form within the compartments, and the quantities of copper sulphate transmitted were irregular and of large value. At present the author is experimenting with the tubes full of a weak gelatine jelly, with the intention, ultimately, of stopping each tube with a short plug of jelly, and thus preventing circulations of all sorts. The plugs will probably be made insoluble with formaldehyde. Mr. Hibbert has helped me with the analyses, and the results are hopeful; but, as already indicated, progress is inevitably slow.

University College; Sheffield.

LI. The Application of an Interforence-Method to the Inrestiuation of Young's Modulus for Wires, and its Relation to Chaniles of Temperature and Magnetization; and a further Applieation of the same. Method to the Study of the Change in Dimensions of Jron and Steel Wires by Maynetizition. By G. A. Shakespear, 1851 Science Scholar, Trinity Colle.je', Cambridge*

M UCH work has been done with the object of elucidating 1 the relation between temperature and elasticity, and more remains to be done.

The problem presents many difficulties, not a few of which arise from the fact that it is not easy to mintain at a constant temlerature, more or less high, a sufficient length of the material under investigation. Again, very slight differences of temperature between the wire undergoing traction and the scale on which the alteration of length is measured may easily lead to erroneous results.

These difficulties may be lessened by having the length of wire smali; but this involves the necessity of some means of measuring accurately rery small changes of length; interference suppli's such a means. In the present work it has not been found necessary to go to a higher order of accuracy than ${ }_{10}^{1}$ of the distance hetween successive interference-bands of sodium light, which represents a length of a bout $0000003 \mathrm{~cm}$.,

* Communicated by Pruf. J. J. Thomson, F.R.S. 